\title{
Wireless Network for Self-Reconfigurable Hardware Nodes
}

\author{
Mikael Heil ${ }^{1}$, Camel Tanougast ${ }^{1}$, Kevin Cheng $^{2}$ and Abbas Dandache ${ }^{1}$ \\ ${ }^{1}$ ASEC-Laboratory of Design, Optimize and Modelization of Systems (LCOMS) University of Lorraine, Metz, France \\ ${ }^{2}$ Institute of Nanotechnologies Ecole centrale de Lyon, Lyon, France \\ \{Mikael.Heil, Camel.Tanougast\}@univ-lorraine.fr
}

\begin{abstract}
In this paper, a model is proposed to perform the Self-Organized Systems (RSS) by using the reconfigurable hardware architectures in a Wireless Sensor Network (WSN) environment. The evaluation performance provide information on the utilization of auto-reconfigurable architecture in WSN using the ZigBee Protocol. The proposed system is designed to study at each level, parameters that influence performance. Thereby, the evaluation platform is described in order to provide data on expected performance and finally the timing analysis shows the limitation in terms of networked computation.

Index Terms - FPGA, WSN, Self-organized system, Zigbee.
\end{abstract}

\section{Introduction}

The evolution of networked systems based selforganization concepts are studied with the objective to give more autonomy and computing power [1]. The Selforganization can be describe as a dynamical and adaptive process where systems acquire and maintain structure themselves, without external control [2]. Therefore, selforganized systems are developed and used with the idea that a group of embedded systems can have the autonomy to interact and work without external influences. Indeed, the networked system has the advantage to connect a huge number of nodes which can evolve and execute tasks in any case. Nodes can be added or removed. Then, the deployment of self-organized structure can be used to make the system fault tolerant in the sense that tasks can be share in the network and if some nodes break down, then tasks can be relocated in other nodes.

In this work, we combine the advantage of reconfigurable hardware and the concept of self-organization to created a structure that can evolve in the time and in the space. More specifically, FPGA and reconfiguration capabilities [3], total or partial, allows us to build hardware systems that can be modify at run-time. In practice, the objective is to realize a networked system composed of nodes that are intelligent, selforganized, auto-reconfigurable and able to work in cooperation with other nodes without external control. The realization of such adaptable capabilities in a networked system introduces problems based on system intelligence and communication. It is the key to create hardware modules that can be relocated on an available reconfigurable system at runtime. An approach concerning self-organization based on intelligent networked system is proposed with a structure for communication. At the network level, communication problems are critical because a network is something which evolves permanently. Nodes are connected and disconnected all the time, and that has an impact on the utilization of all provided functionalities. In this context, a way to share hardware resources is observed and the required communication structure is studied under the form of the association of self-organization and FPGA technology using partial reconfiguration techniques [4].

In this paper, we propose an association of AutoReconfiguration and Self-Organization under the form of Reconfigurable Self-Organized Systems (RSS). This association is defined as follows: A RSS is a modular and complex system based on reconfigurable hardware technology. It is able to organize and restructure its own activity without external influences, but by the interactions of its intelligent nodes (defined as Reconfigurable System Nodes - RSNs) [1]. Essential resources that must be available on each node of a distributed cooperative system are minimal local computation power, resources management, the ability to communicate and distribute or gather tasks in the network. We focus here on the decentralization of the distribution tasks where the nodes have the autonomy to work spontaneously together. The advantage is that nodes are not depending of a central command node, because if this node break down then the system cannot work anymore.

This paper is organized as follows. Section 2 describes the context of the utilization of wireless medium as communication link for the Self-organized reconfigurable System. Section 3 presents the model used to implement the system and the platform which is realized. This section presents the designed platforms that are used to implement and test the proposed concept. Section 4 shows the time measurement methodology and observed performance. Finally, conclusions are given in Section 5.

\section{Auto-Reconfiguration Self-organized Wireless Sensors Network}

To realize the self-organization of intelligent reconfigurable nodes in a network, the communication system should be design to be integrated into the standard communication architecture. The first part is used for the tasks distribution using a request stream that is build to broadcast a request for help other nodes. The second part is used to transfer data and bitstream files. In general, a task is represented by a pack of one partial bitstream and data. This pack can be sent over a network to be processed by any intelligent node. The idea is to reverse the process of tasks 
distribution. Therefore, only available nodes reply and by consequence, free hardware resources are detected. In this condition, each node is free to reply whenever they want and a group can be created spontaneously to reply to a demand. In this context, the best communication structure is a broadcasting scheme where nodes send requests to all others nodes at the same time. In practice and to reduce the complexity, it is choose to create a stream that contains requests and which travels from node to node in a network where the number of nodes is known. The second part of the communication is established when nodes reply to accept tasks. Then, a bidirectional transaction is managed to exchange bitstreams and data. The starting point to realize such intelligent network is the design of intelligent node which is the subject of the next section.

Wireless Sensor Networks (WSNs) are becoming more and more important and they can gain advantage of reconfigurable technology, in terms of flexibility, energy consumption and sensor lifetime. Indeed, a Wireless Sensor Network (WSN) provides different aspects the sharing information [5]. In parallel, the Zigbee networks have been optimized allowing it to reconfigure itself improving flexibility and forming network strategy [6]. For example, this reconfigurable process can be useful to dynamically organize the network to transmit through obstacle present in the transmission field. This example shows the reconfiguration capacity at the network level. It is the base which prefigure the utilization of reconfigurable technology into WSN and it is the origin of the presented implementation which has the objectif to experiment and discuss this combination. Indeed, as reconfiguration capacity is becoming crucial for future electronic design, systems, and networks, studying their impact on nodes that composed those WSN can impact the development process of WSN nodes. By giving to these nodes an auto-reconfiguration capacity, we contribute to the domain by upgrading to node's potential. For instance, the faulttolerance capacity is improved at node level as well as network level.

ZigBee wireless communication technology is mainly characterized by a high resistance to noise, low power devices, low bit rate, and low cost. ZigBee offers specifications more and more popular support for sensors networks which require low bit rate communication such as Wireless Sensor Networks (WSNs) [6]. These network sensor nodes can sense, measure, and gather information from the environment and based on some local processes. Therefore, they can transmit the sensed data to the user. WSNs can be classify into five groups (terrestrial, underground, underwater, multi-media and mobile) which have theirs own characteristics [5]. Researches in Multimedia WSN are mainly focused on high bandwidth demand, high energy consumption and quality of service (QoS) of system enabling monitoring and tracking of multimedia such as video, audio, and imaging. The proposed system using RSS and ZigBee enters in the field of multi-media WSN and it is presented in the following section. The auto-reconfiguration is the capacity of a system to reconfigure itself during runtime and without external influence. In such design, a System on Chip (SoC) is implemented in a reconfigurable FPGA, and it includes a controller used to communicate, reconfigure the partial reconfigurable region (Interface Configuration Access Port-ICAP) and manage the resulting data [7-14]. Therefore, partial-reconfiguration technology allows a node (RSN) to be auto-reconfigurable. Consequently, an exploration of important parameters of reconfigurable systems and their impact on performance, power dissipation, and energy consumption for different application scenarios can be study. The proposed concept of Reconfigurable Self-Organized System intends to allows networked structure to manage its own tasks and hardware resources (in particulars reconfigurable modules).

\section{Wireless model of Reconfigurable Self-organized System}

\section{A. Wireless Model}

The Wireless model describes the utilization of autoreconfiguration capability to provide fault-tolerance capacity and higher hardware module performance to sensors network. RSNs are able to reconfigure themselves on the fly and to exchange configuration and data files. Consequently, a fault tolerance capacity can be established in order improvement the life-time of each nodes and keep the running processing viable. Figure 1 presents the model of nodes where each are composed of an FPGA based board and a XbeePro device enabling ZigBee Pro communication. In this model, each node is dynamic and autonomous making the networked group able to work without any master node or central node.

\section{B. Evaluation Platform}

The evaluation platform node is mainly composed of two FPGA platforms. The first one is a Xilinx ML507 board which embed a FPGA Virtex 5FX70 associated with a XBee module [15]. The second platform is a Digilent Nexys 3 board (FPGA Spartan 6) which is used to send tasks and reconfigurable module like a Dynamic, Autonomous and Interoperable (DAI) node can do. Finally, a ZigBee Sniffer is used to analyze the ZigBee network [16]. Figure 2 shows the demonstration platform used to study the influence of auto-reconfiguration in a WSN.

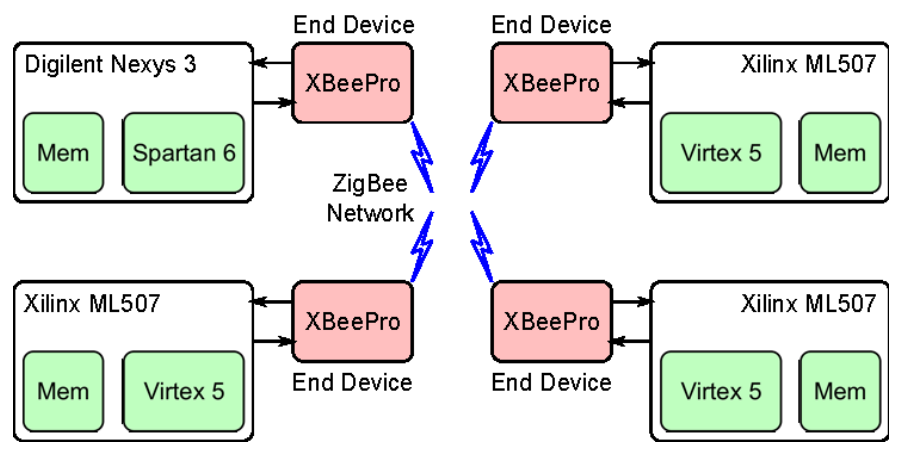

Fig. 1 Network model using reconfigurable platforms. 


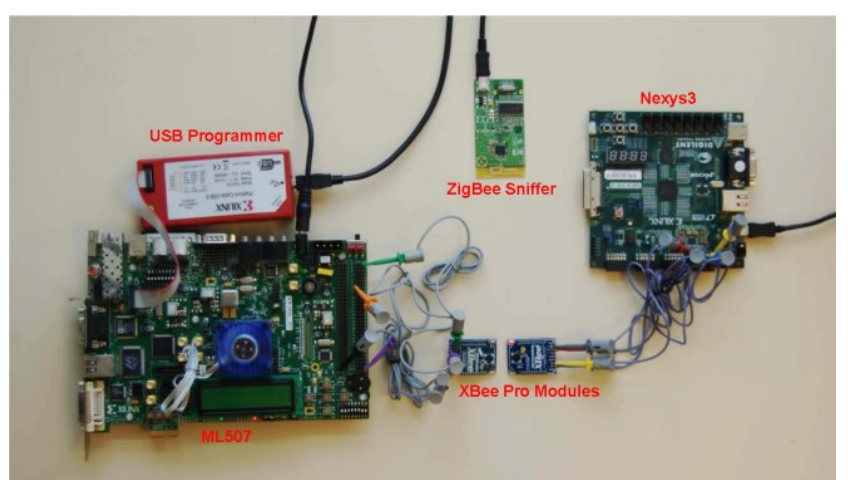

Fig. 2 The demonstration platform.

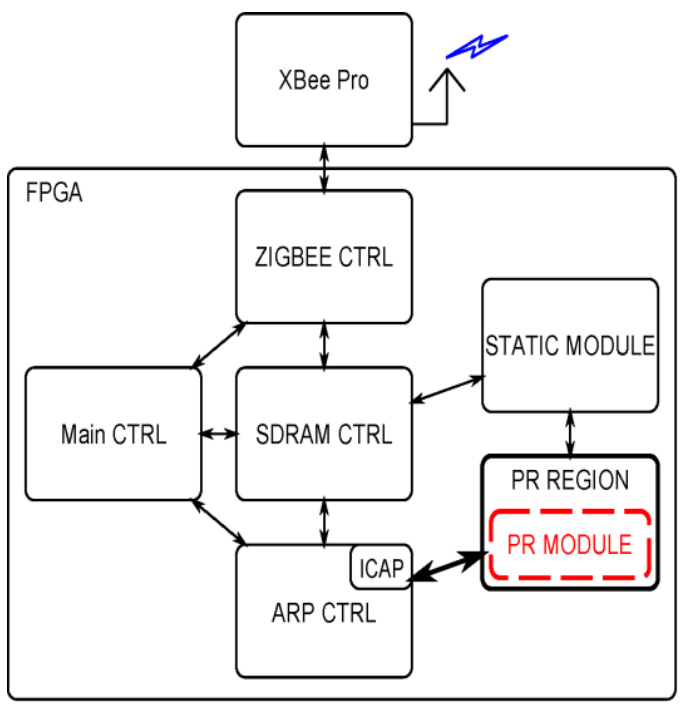

Fig. 3 Hardware architecture of a RSN.

1) System on FPGA Architecture: Figure 3 presents the hardware architecture of the RSN implemented in FPGA used for the platforms. It is both used for the selected Digilent Nexys3 and the Xilinx ML507 boards.

The RSN architecture is completely developed in a VHDL structural description. It is mainly composed of five modules. Three IPs are designed to control the XBee Pro module, the SDRAM memory and the ICAP. In addition, the main controller is used to manage the all process. Concerning the application, a black and white filter is used as a common example. It allows to validate the utilization of autoreconfiguration as described over the ZigBee wireless sensors network.

2) ZigBee Configuration: ZigBee modules can be configured among four different types including (see Fig. 4):

- Coordinator

- Router

- Full Function Device

- Reduce Function Device

In the case of the demonstration platform presented here, all ZigBee modules are configured as Full Function Device.
The ZigBee network is built from Xbee Pro devices configured as Reduced Function Device.

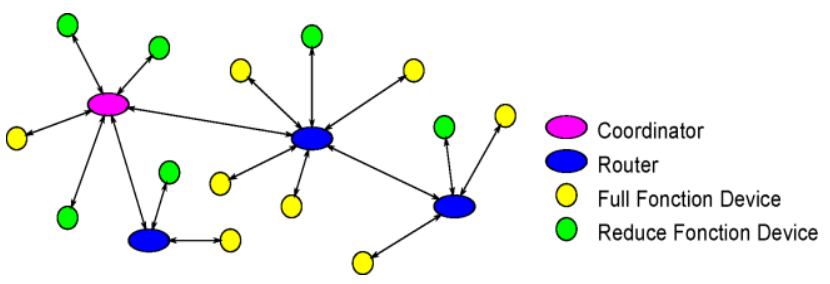

Fig. 4 ZigBee different device types.

Using this configuration, each device can transmit information in a broadcast mode which include an identifier in the transmitted frame. It allows to implement the node identifier management in controller of the Reconfigurable node.

3) Communication Protocole: The communication protocol used in the evaluation platform is using a simple broadcast over the ZigBee link to request assistance from another node of the network. Then, a simple hand shake protocol to associate the node which require help to process the image and the node which has free hardware resources. As the transaction is finished, the two nodes are able to exchange files using the ZigBee link.

\section{Timing analysis and Performance}

Figure 5 shows the result of the black and white filter used as demonstration application. The timing analysis shows a limit in the utilization of auto-reconfiguration to increase performance of a Wireless Sensor Network using Zigbee Communication. Table I presents the time request at each stage of the processing in the organization of the relocalization of a task over the network. As the time request to transmit the files are in the magnitude order of the second, the time required to reconfigure the PR-region about the millisecond. This is the limit for distributed computing system. The performance measures of the system are based on the system presented earlier. The measures show that file transmission time is in the magnitude order of the second with is a drawback in terms of computing performance. Nevertheless, it is also an advantage considering the fault tolerance capacities. In such case, spend only a few seconds to save the current tasks from being lost is acceptable. In addition, the well know possibility to partially reconfigure the FPGA blank bitstream is a main advantage in term of computation power which a main drawback in the case of WSN applications.

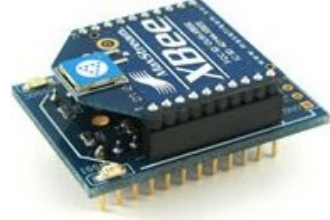

Origina 1 Image

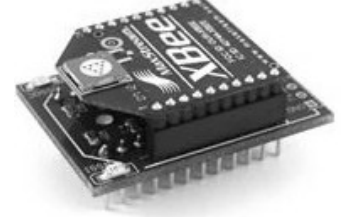

Filtered Image
Fig. 5 Image processing results performed by the relocated wireless node. 
TABLE I Results of the Timing Measurement.

\begin{tabular}{|c|c|c|c|c|c|}
\hline State & $\begin{array}{c}\text { Size } \\
\text { (in bytes) }\end{array}$ & $\begin{array}{c}\text { Estimated } \\
\text { Times }\end{array}$ & $\begin{array}{c}\text { Simulated } \\
\text { Times }\end{array}$ & $\begin{array}{c}\text { Measured } \\
\text { Times }\end{array}$ & Comments \\
\hline \hline $\begin{array}{c}\text { Filtering } \\
\text { (Nexys) }\end{array}$ & 82398 & 0.41199 & 0.033378 & 0.034 & $\begin{array}{c}\text { Processing on } \\
\text { Nexys3 }\end{array}$ \\
\hline $\begin{array}{c}\text { Send } \\
\text { bitstrea } \\
\text { m }\end{array}$ & 66946 & 5.811 & 6.561 & 6.562 & $\begin{array}{c}\text { From Nexys3 } \\
\text { to ML507 }\end{array}$ \\
\hline $\begin{array}{c}\text { Send } \\
\text { Image }\end{array}$ & 82398 & 7.153 & 8.076 & 8.076 & $\begin{array}{c}\text { From Nexys3 } \\
\text { to ML507 }\end{array}$ \\
\hline $\begin{array}{c}\text { Reconfig } \\
\text { uration }\end{array}$ & 66946 & 0.006696 & 0.007359 & 0.007359 & $\begin{array}{c}\text { ML507 } \\
\text { Capacity }\end{array}$ \\
\hline $\begin{array}{c}\text { Filtering } \\
\text { (ML) }\end{array}$ & 82398 & 0.041199 & 0.041755 & 0.042 & $\begin{array}{c}\text { Processing on } \\
\text { ML507 }\end{array}$ \\
\hline $\begin{array}{c}\text { Send } \\
\text { Result }\end{array}$ & 82398 & 7.153 & 8.809 & 8.81 & $\begin{array}{c}\text { From ML507 } \\
\text { to Nexys3 }\end{array}$ \\
\hline
\end{tabular}

\section{Conclusion and Future Works}

This paper proposes the hardware implementation of a reconfigurable self-organized wireless nodes network performing the Self-Organized concept. The study of the communication and the reconfiguration of a network, and the reconfiguration of intelligent nodes is the main challenge of RSS and specially of the Hardware links. For this purpose, a model is proposed to perform the Self-Organized Systems (RSS) by using the reconfigurable hardware architectures in a Wireless Sensor Network (WSN) environment. The approach shows the influence of the existing technology and the selforganized context. Indeed, auto-reconfiguration is main characteristic of a dynamic, autonomous and interoperable system offering fault tolerant capacity, low power consumption, and computation power. In the context of the sensors network using ZigBee communication, this autoreconfiguration is a true benefit in the functionality of the nodes that compose the system. The real time measurement results are also given. In spite the drawback concerning the communication delays, we can conclude on the positive benefit of deploying system able to exchange they tasks and hardware resources in such specific WSN. Some details concerning the implementation and the development of properties related with self-organization, fault tolerance and video processing are pointed. Future works include implementation of heterogeneous system including different logical resources, and the development concerning the links between distributed and self-organized systems.

\section{References}

[1] K. Cheng, A. A. Zarezadeh, F. Muhlbauer, C. Tanougast, and C. Bobda, "Auto-reconfiguration on self-organized intelligent platform," in Proceedings of Adaptive Hardware and Systems (AHS), 2010 NASA/ESA Conference on, vol. 2010, pp. 309-316, IEEE, 2010.

[2] K. Cheng, C. Tanougast, C. Bobda, and A. Dandache, "Peer-to-peer control application for reconfigurable self-organised system," in Proceeding of Signal and Image Processing Conference 2011 (K. Rao, ed.), vol. 2011, ACTA Press, 2011.

[3] Xilinx, "Virtex-5 fpga configuration user guide," Xilinx, vol. 2008, 2008.

[4] Xilinx, "Early access partial reconfiguration user guide," Xilinx, vol. 2008, 2008.

[5] J. Yick, B. Mukherjee, and D. Ghosal, "Wireless sensor network survey," Computer Networks, vol. 52, no. 12, pp. 2292-2330, 2008.

[6] Y. XU, S. QIU, and M. HOU, "Reconfigure zigbee network based on system design," Wireless Sensor Network, vol. Volume: 01, pp. Pages: 206-211, 2009.

[7] M. Birk, C. Hagner, M. Balzer, N. V. Ruiter, M. Hubner, and J. Becker, "Evaluation of the reconfiguration of the data acquisition system for $3 \mathrm{~d}$ usct”, International Journal of Reconfigurable Computing, vol. 2010, p. 9 pages, 2010 .

[8] B. Glas, O. Sander, V. Stuckert, K. D. Muller-Glaser, and J. Becker, "Prime field ecdsa signature processing for reconfigurable embedded systems," International Journal of Reconfigurable Computing, vol. 2010, p. 12 pages, 2010.

[9] D. Gohringer, T. Perschke, M. Hubner, , and J. Becker, "A taxonomy of reconfigurable single-/multiprocessor systems-on-chip," International Journal of Reconfigurable Computing, vol. 2008, p. 11 pages, 2008.

[10] D. Gohringer, J. Obie, A. L. S. Braga, M. Hubner, C. H. Llanos, and J. Becker, "Exploration of the power-performance tradeoff through parameterization of fpga-based multiprocessor systems," International Journal of Reconfigurable Computing, vol. 2010, p. 17 pages, 2010.

[11] D. Gohringer, M. Hubner, E. N. Zeutebouo, and J. Becker, “Operating systemfor runtime reconfigurable multiprocessor systems," International Journal of Reconfigurable Computing, vol. 2010, p. 16 pages, 2010.

[12] C. Schuck, B. Haetzer, and J. Becker, "An interface for a decentralized $2 \mathrm{~d}$ reconfiguration on xilinx virtex-fpgas for organic computing," International Journal of Reconfigurable Computing, vol. 2008, p. 11 pages, 2008.

[13] C. Schuck, B. Haetzer, and J. Becker, "Reconfiguration techniques for selfx power and performance management on xilinx virtex $2 /$ virtex 2 pro fpgas," International Journal of Reconfigurable Computing, vol. 2010, p. 12 pages, 2010.

[14] A. Klimm, B. Glas, M. Wachs, S. Vogel, K. D.Muller-Glaser, and J. Becker, "A security scheme for dependable key insertion in mobile embedded devices," International Journal of Reconfigurable Computing, vol. 2010, p. 19 pages, 2010.

[15] Digi, "Xbee/xbee-pro rf modules, product manual v1.xex - 802.15.4 protocol," Digi International Inc, vol. 2009, 2009.

[16] Microchip, "Zena wireless network analyzer, users guide, reference ds51606c," Microchip Technology, vol. 200, 2008. 